\title{
Short communication: Methicillin-resistant Staphylococcus aureus in bulk tank milk of dairy cows and effect of swine population density
}

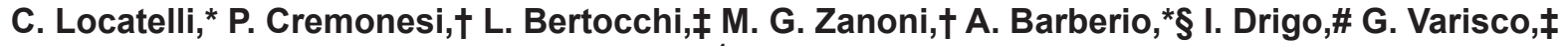 \\ B. Castiglioni, $\dagger$ V. Bronzo, ${ }^{*}$ and P. Moroni* $\|^{1}$ \\ *Dipartimento di Scienze Veterinarie per la Salute, la Produzione Animale e la Sicurezza Alimentare, Università degli Studi di Milano, \\ 20133 Milan, Italy \\ †Istituto di Biologia e Biotecnologia Agraria, Consiglio Nazionale delle Ricerche, via Einstein, 26900 Lodi, Italy \\ ‡lstituto Zooprofilattico Sperimentale della Lombardia e dell'Emilia-Romagna, via Bianchi 9, 25124 Brescia, Italy \\ §Istituto Zooprofilattico Sperimentale delle Venezie, Sez. terr. Vicenza viale Fiume 78, 36100 Vicenza, Italy \\ \#Istituto Zooprofilattico Sperimentale delle Venezie, Sez. terr. Treviso, V.le Mazzini 4, 31020 Villorba (TV), Italy \\ IIAnimal Health Diagnostic Center, Quality Milk Production Services, Cornell University, Ithaca, NY 14853
}

\section{ABSTRACT}

The methicillin-resistant Staphylococcus aureus (MRSA) has recently frequently been reported in dairy cattle, usually with low prevalence. The livestockassociated MRSA (LA-MRSA) ST398 is especially involved in cases of subclinical and clinical mastitis. Swine carry LA-MRSA without clinical symptoms and are considered its reservoir and shedder. People exposed to swine are particularly at risk of LA-MRSA colonization. Environments with relevant livestock density are a demonstrated risk factor for humans to be carriers of a LA-MRSA. This work investigated dairy farms located in an area with a high livestock density, mainly represented by swine. Bulk tank milk samples from 224 dairy farms were collected, and their status was defined as MRSA-positive or MRSA-negative based on culture on chromogenic medium. The number of fattening swine and of fattening swine herds was calculated in an area of $3 \mathrm{~km}$ around each dairy farm through georeferencing. The probability of a Staphylococcus aureus-positive dairy farm to be MRSA positive based on the extent of potential infective pressure due to swine density was calculated. Both the number of swine herds and the number of swine were associated with the MRSA status of dairy herds. The 9 MRSA isolated were typed by multi-locus sequence typing and spa-typing, and characterized for their virulence factors and antimicrobial resistance profiles. The ST and $s p a$-types detected are consistent with those present in the Italian swine population. Virulence and resistance profiles are mostly consistent with the types detected.

Received June 10, 2015.

Accepted November 7, 2015.

${ }^{1}$ Corresponding author: paolo.moroni@unimi.it
This work provides the first evidence of the epidemiological challenge exerted by the density of the swine population on MRSA in dairy cows.

Key words: methicillin-resistant Staphylococcus aureus, bulk tank milk, cow, swine density, georeferencing

\section{Short Communication}

Methicillin-resistant Staphylococcus aureus (MRSA) has been a public health concern since the 1960s, when strains linked to healthcare settings and nosocomial infections (hospital-acquired MRSA) were reported. In the 1990s, other MRSA strains started circulating among the general population without history of admission to healthcare facilities (community-acquired MRSA; DeLeo et al., 2010). The emergence of livestock-associated strains (LA-MRSA) made MRSA an animal health challenge as well (Voss et al., 2005). Some occupational categories (veterinarians, farmers, slaughterhouse employees) are particularly at risk to be MRSA nasal carriers (van den Broek et al., 2009; van Cleef et al., 2011) and may develop infections due to the contact with food animals and the farm environment. The spread and persistency of the LA-MRSA ST398 in particular in swine production chains has been described, and the role of environmental dust as a MRSA vector and reservoir has been clarified (EFSA, 2010; Broens et al., 2011; Friese et al., 2012; Merialdi et al., 2013). A correlation between the detection of LA-MRSA ST398 in humans and the density of swine and cattle population was observed (van Loo et al., 2007). Bulk tank milk (BTM) represents an excellent and increasingly used herd-level screening tool for the detection of contagious mastitis pathogens. Several surveys have been carried out using BTM culture to detect MRSA in dairy farms (Haran et al., 2012; Kreausukon et al., 2012; Paterson et al., 2012), revealing a prevalence with relevant regional differences ranging from $0.3 \%$ in the United Kingdom 
(Paterson et al., 2012) to $4.4 \%$ in Germany (Kreausukon et al., 2012). Apart from different sampling choices and different laboratory methods and media used, it is conceivable that many factors associated with the studied population and the epidemiological background of the area, including farm density and mutual position, could contribute in determining the MRSA prevalence.

Global positioning systems and geographic information systems have become effective tools for the comprehension of infectious diseases epidemiology and dynamics (Feingold et al., 2012; Smit et al., 2012). The farms were localized in an area with a very high density of livestock farming, particularly with a high density of swine holdings. The aim of this study was evaluate the association between MRSA in BTM of dairy herds and the density of swine and swine holdings around each dairy farm and to type the MRSA isolates. The study area included part of 3 Northern Italy provinces, Brescia, Bergamo, and Mantova (Figure 1), where Italian dairy and swine farms are very concentrated. Once a year, all dairy farms located in Brescia province or delivering milk to dairy industries in Brescia are controlled through BTM culturing for the presence of Staphylococcus aureus and Streptococcus agalactiae. From February to March 2011, BTM samples were collected from 224 dairy herds that resulted positive for $S$. aureus at the previous annual screening, out of a total of 1,102 controlled farms. Once thawed at room temperature, a 2-step enrichment protocol was applied to $1 \mathrm{~mL}$ of each BTM sample for MRSA detection as previously described (Spohr et al., 2011). Finally, $100 \mu \mathrm{L}$ of the inoculated TSB broth was spread onto MRSA Chromogenic Agar (Laboratorios Conda, Madrid, Spain) and the plates were incubated for $48 \mathrm{~h}$ at $37^{\circ} \mathrm{C}$. Bluegreenish colonies with halo were considered putatively MRSA, picked up, and subcultured. For MRSA confirmation, a duplex PCR, which detected nuc (Baron et al., 2004) and mecA (Murakami et al., 1991) genes, was performed. A third PCR was performed targeting the variant mecC gene according to Paterson et al. (2012). Herds were classified in 2 categories, referred to as "status" hereafter throughout the paper: (1) MRSAnegative herds; (2) MRSA-positive herds.

The SCC was determined for all BTM samples by an automated somatic cell counter (Bentley Somacount 150, Bentley Instruments, Chaska, MN). Multilocus sequence typing was performed according to Enright et al. (2000) with minor modifications, and multi-locus sequence type $(\mathbf{S T})$ was assigned based on sequence allelic profiles using the multi-locus sequence typing (MLST) database website (http://www.mlst.net). The $\mathrm{X}$ region of the spa gene was amplified according to Shopsin et al. (1999). To obtain the spa type, the sequences were analyzed with the Ridom StaphType soft- ware program (version 1.4; Ridom, GmbH, Wurzburg, Germany, http://spa.ridom.de/index.shtml). The DNA was amplified to verify the presence of virulence-associated genes using the panel of primers and protocols described by Cremonesi et al. (2013). The virulence factors considered were thermonuclease encoded by the nuc gene, and the enterotoxins A, B, C, D, E, G, H, I, J, K, and L encoded, respectively, by sea, seb, sec, sed, see, seg, seh, sei, sej, sek, and sel genes; the clumping factor A, toxic shock syndrome toxin 1, and exfoliative toxins $\mathrm{A}$ and B encoded, respectively, by clfA, tsst, eta, and $e t b$ genes. The presence of the following virulence factors was also investigated: leukocidin $\mathrm{E}$ (lukE), leukocidins $\mathrm{E}$ and $\mathrm{D}$ (lukE-lukD), Panton-Valentine leukocidin $(l u k F / S-P V)$, chemotaxis inhibitory protein $(c h p)$, fibronectin binding protein $(f m t B)$, staphylokinase $(s a k)$, staphylococcal complement inhibitor (scn), and collagen binding protein ( $c n a)$.

Minimum inhibitory concentrations of 20 antibiotics were determined for each MRSA isolated, using broth dilution test, according to the procedure described in CLSI guidelines Vet01A4 (CLSI, 2013a). The antimicrobials tested were ampicillin, oxacillin $+2 \%$ $\mathrm{NaCl}$, amoxicillin/clavulanic acid, amikacin, cefoxitin, ticarcillin, cefpodoxime, ticarcillin/clavulanic acid, trimethoprim/sulfamethoxazole, cefazolin, gentamicin, imipenem, clindamycin, penicillin, doxycycline, ceftiofur, marbofloxacin, rifampin, enrofloxacin, and erythromycin. A commercially available microdilution MIC system (Sensititer compan1F, Trek Diagnostics Systems, East Grinstead, UK) was used according to the manufacturer's instructions. Antimicrobial sensitivity was evaluated according to the breakpoints listed in CLSI Vet01-S2 guideline for Staphylococcus spp. (CLSI, 2013a). Disk diffusion test was performed according to the procedure described in CLSI guidelines Vet01A4 (CLSI, 2013a) for cefoxitin as a marker for oxacillin and methicillin resistance, according to CLSI guidelines (CLSI, 2013b). Staphylococcus aureus ATCC 29213 was used as a quality control strain for both the test, MIC and disk diffusion.

All fattening swine holdings in the same area were also localized within a $3 \mathrm{~km}$ radius from each positive and negative dairy herd (Figure 1). This distance was chosen considering those used by Smit et al. (2012) for Q fever and taking into account that operations associated with farms (e.g., livestock transportation, manure spreading) contribute to MRSA diffusion. The number of swine herds and the average size of swine herds were used to calculate animal density within the traced area around each dairy farm. The choice of fattening swine herds is based on the results of Friese et al. (2012) that found a much higher frequency of MRSA-positive dust samples in this type of farm than in breeding farms. 
All statistical analyses were performed using SPSS 22.0 (IBM, SPSS Inc., Chicago, IL). For statistical purposes, bulk milk SCC were transformed in somatic cell linear score (BMSCS) to normalize their distribution as described by Kirk (1984). The BMSCS, number of herds, and animal density, both for dairy farms and fattening swine farms, did not follow a normal distribution according to Shapiro-Wilk test. Difference between BMSCS in negative and positive MRSA S. aureus dairy farms was assessed by a nonparametric test (U MannWhitney) as the average number of lactating cows and fattening swine herds number and their density. A binary logistic regression analysis was used to evaluate the probability for a dairy farm with $S$. aureus-positive BTM, of being also MRSA-positive, related to the fattening swine herds number and density present in a 3 $\mathrm{km}$ area around the farm. Statistical significance was accepted at $P<0.05$.
Putative MRSA colonies (up to 5 per suspected plate) were picked up from chromogenic agar and tested for confirmation as described above. In 9 BTM samples MRSA isolates were detected and confirmed. All isolates were positive for mecA and negative for mecC gene. Considering all the herds tested, the prevalence of MRSA-positive herds found was $4 \%$. The 9 MRSA-positive isolates were genotyped and characterized (Table 1). Two multilocus ST were detected: ST398 $(\mathrm{n}=5)$ and ST97 $(\mathrm{n}=4)$. Three spa types were detected belonging to ST398: $\mathrm{t} 899(\mathrm{n}=3)$, t001 $(\mathrm{n}=1)$, and t108 $(\mathrm{n}=1)$. The spa types belonging to ST97 were $\mathrm{t} 4795$ (n $=3)$ and $\operatorname{t9305}(\mathrm{n}=1)$. All the isolates were negative for PVL, toxic shock syndrome toxin, and staphylococcal enterotoxins. The genes coding for staphylokinase, staphylococcal complement inhibitor, and chemotaxis inhibitory protein were also undetected. The MIC confirmed resistance of all the MRSA to doxycycline,

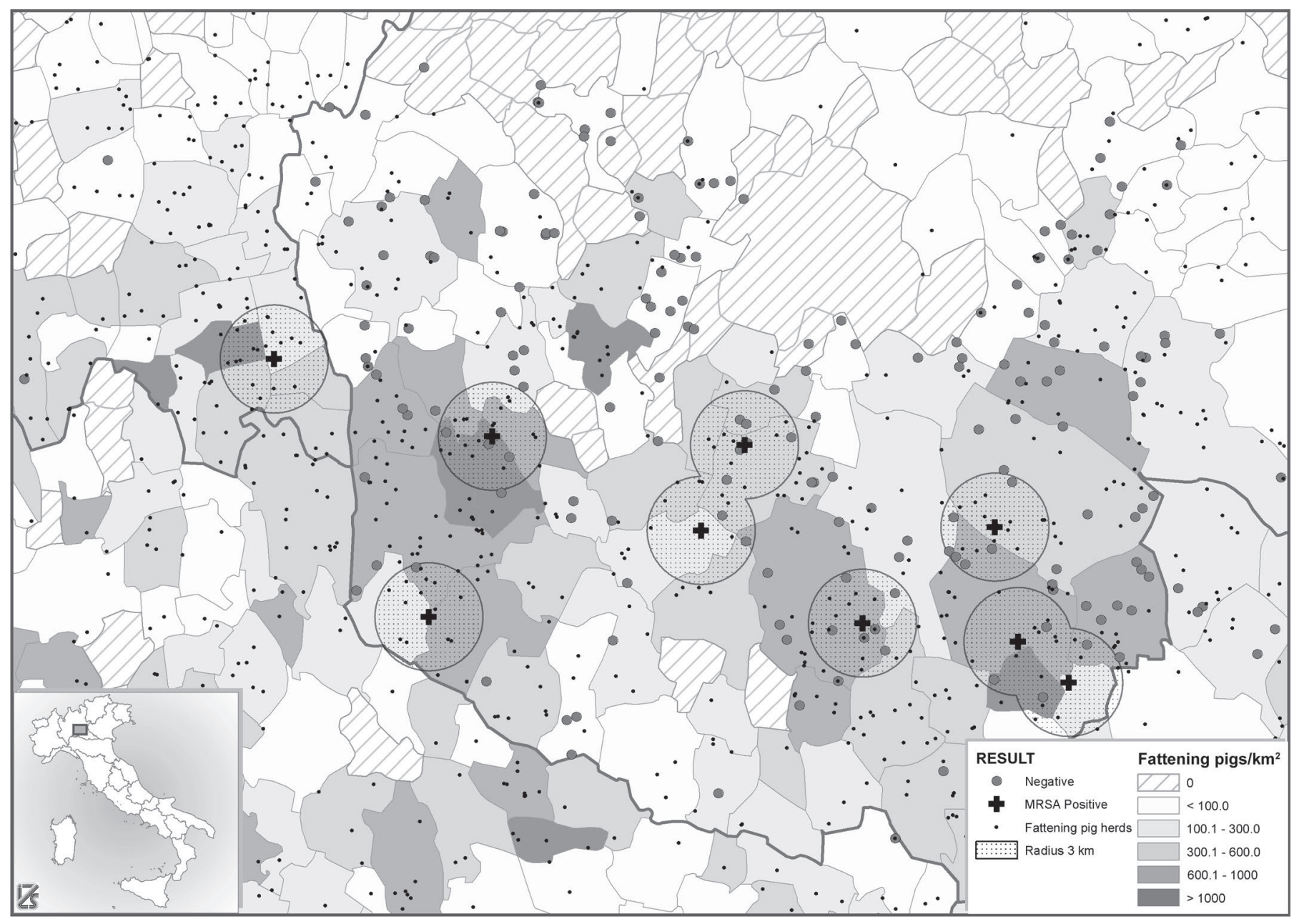

Figure 1. Spatial distribution of the epidemiological elements involved in a map of the study area. Grey dots represent the negative samples, and black crosses represent methicillin-resistant Staphylococcus aureus (MRSA)-positive dairy farms. The black dots are the fattening swine herds. The different grey intensities represent the density of swine in each municipality. 
penicillin, and ampicillin. All 9 isolates were susceptible to trimethoprim/sulfamethoxazole. Resistance rate was $22 \%$ for amikacin, $78 \%$ for gentamicin and erythromycin, $89 \%$ for clindamycin, marbofloxacin, and enrofloxacin, $55 \%$ for ceftiofur. According to MIC results, 3 strains out of $9(2785,2605$, and 2795) were susceptible to oxacillin ( $\mathrm{MIC} \leq 2 \mu \mathrm{g}$ ) and cefoxitin ( $\mathrm{MIC} \leq 4 \mu \mathrm{g}$ ), and another (2601) displayed resistance only against cefoxitin (with the MIC for oxacillin $=2 \mu \mathrm{g}$ ).

The average number of lactating animals per herd was 208.9 cows $(\mathrm{SD} \pm 191.6)$, with a minimum of 4 and a maximum of 1,320 . The average size of dairy herds tended to be positively associated with MRSA status $(P=0.08)$. The bulk milk SCC geometric mean was numerically higher in MRSA-positive dairy herds, but the value was not significant $(286,000 \pm 212,000$ vs. $236,000 \pm 231,000, P=0.38)$. The number of fattening swine herds in the traced $3 \mathrm{~km}$ area was significantly higher around MRSA-positive herds $(P<0.001)$ compared with the area surrounding the negative dairy herds. Similarly, the swine population in each of the 3 $\mathrm{km}$ areas was significantly higher around MRSA-positive herds $(P<0.001)$ compared with the area around the MRSA-negative dairy herds.

The binary logistic regression model demonstrated a significant association between the number of fattening swine herds $(P<0.001)$ and the estimated probability for a $S$. aureus-positive dairy farm of being MRSA positive. Similarly, the number of swine was also associated $(P<0.001)$ with the probability of MRSA positivity.

This study reports an evaluation of MRSA prevalence in Italian dairy herds based on BTM samples in an area with high density of dairy cows and swine. Although evaluated at a local level, the low prevalence recorded is in agreement with data available from similar studies (Kreausukon et al., 2012; Haran et al., 2012; Paterson et al., 2012). Recent work that sampled dairy cows at quarter level showed a low prevalence in Italian dairy herds in different regions (Luini et al., 2015). The disagreement with the significantly higher rate found from Antoci et al. (2013) is probably due to the selection of sampled herds among those that already experienced MRSA in the previous $3 \mathrm{yr}$ and to variability in the amount of samples and in testing methods. To the authors' knowledge, this is the first statistical demonstration of the effect of swine population density on dairy cattle LA-MRSA status at milk level. Tavakol et al. (2012) detected MRSA ST398 in 14 dairy herds in the Netherlands and stated that the fact that 9 of them (64\%) harbored both cows and swine was a confirmation of MRSA transmission among animals.

The lineages detected were those prevalent in a swine population that is mainly concentrated in the area where the current study was performed, or in areas with

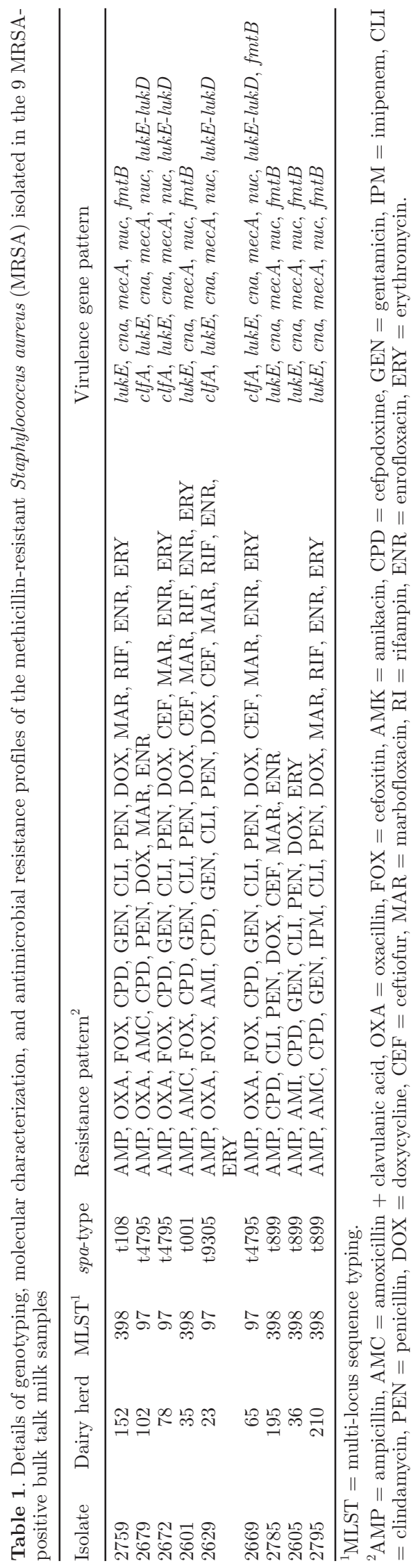


comparable epidemiological features (Battisti et al., 2010). The only exceptions were t001 and t9305. Moreover, the presence of multiple ST and spa types mirrors the heterogeneity reported by Battisti et al. (2010). The virulence gene patterns (Table 1 ) were consistent with the ST and spa types. Isolate 2669 was the only exception as it was positive for the fibronectin binding protein gene encoding the fibronectin binding protein, unlike the other isolates belonging to ST97 t4795. The absence of virulence genes usually detected in human $S$. aureus is in agreement with previous studies (Battisti et al., 2010; Kreausukon et al., 2012; Molla et al., 2012; van Duijkeren et al., 2014) and contributed to exclude a human to animal transmission of the present isolates (Nemeghaire et al., 2014). The ST97 belongs to the clonal complex CC97 that is one of the most prevalent lineages in Italian swine production chain and in bovine mastitis (Battisti et al., 2010; Luini et al., 2015). Spoor et al. (2013) recently demonstrated that CC97 was of bovine origin and then passed to humans and other animal species, whereas CC398 was of human origin and then passed to swine, acquiring methicillin resistance. The different host association and species-to-species jumps could justify the peculiar virulence gene pattern displayed.

The antimicrobial resistance profiles were quite different within a genotype, denoting a genetic and phenotypic variability among the strains detectable in the population (Feßler et al., 2012). The only isolates with the same pattern of resistance were 2672 and 2669 belonging to ST97 spa type 4795. All the isolates demonstrated resistance to more than 2 antimicrobial classes. The widest antimicrobial resistance profile was that of isolate 2629, belonging to ST97 t9305. In general, the resistance of all the 9 isolates to doxycycline and tetracycline was consistent with that peculiar to LA-MRSA from swine and cows (Battisti et al., 2010; Vanderhaeghen et al., 2010; Spohr et al., 2011; Kreausukon et al., 2012). Conversely, all the isolates were susceptible to trimethoprim/sulfamethoxazole that is uncommon in MRSA from production animals (Vanderhaeghen et al., 2010; Kreausukon et al., 2012; Nemeghaire et al., 2014). All the isolates but 2605 were resistant to fluoroquinolones, and this is a very high prevalence when compared with that observed by other studies (Vanderhaeghen et al., 2010; Kreausukon et al., 2012).

One isolate out of 9 was found susceptible to oxacillin in both the methods used for antimicrobial susceptibility evaluation, and only 5 were resistant to cefoxitin. However, the mecA gene was detected in each of the 9 isolates, so the definition of MRSA provided by CLSI (2013a) was fully satisfied. Moreover, oxacillinsusceptible MRSA has been described (Hososaka et al., 2007; Kumar et al., 2013), and it has been reported as being carried by dairy cattle as well (van Duijkeren et al., 2014).

This is the first work applying georeferencing to the likelihood of dairy farms to be MRSA positive in relation to the swine population density. The findings of this work demonstrated a clear exposure-response relationship between the number of swine and swine herds and each dairy farm's status in regard to methicillin resistance. Environmental dust is a passive spreader of MRSA, carried by the wind and contaminated items (cloths, boots, gloves, and so on), and it is possible that with these dynamics MRSA could spread from swine to nearby dairy farms (Friese et al., 2012; Merialdi et al., 2013). This could have potential implications in surveillance of MRSA in livestock and humans as well. Specifically regarding dairy farms, MRSA transmission, in a MRSA-contaminated environment, should be reduced through milking hygiene and culling of MRSA-positive cows (Spohr et al., 2011).

\section{ACKNOWLEDGMENTS}

The authors thank the technicians who contributed to the work, in particular Antonio Casula, and Marco Tironi for georeferentiation. This work is part of the project "Survey on the risk factors related to the spreading of antimicrobial resistance in production animals" that Università degli Studi di Milano (Milano, Italy) funded as a postdoctoral grant.

\section{REFERENCES}

Antoci, E., M. R. Pinzone, G. Nunnari, S. Stefani, and B. Cacopardo. 2013. Prevalence and molecular characteristics of methicillin-resistant Staphylococcus aureus (MRSA) among subjects working on bovine dairy farms. Infez. Med. 21:125-129.

Baron, F., M. F. Cochet, J. L. Pellerin, N. Ben Zakour, A. Lebon, A. Navarro, I. Proudy, Y. Le Loir, and M. Gautier. 2004. Development of a PCR test to differentiate between Staphylococcus aureus and Staphylococcus intermedius. J. Food Prot. 67:2302-2305.

Battisti, A., A. Franco, G. Merialdi, H. Hasman, M. Iurescia, R. Lorenzetti, F. Feltrin, M. Zini, and F. M. Aarestrup. 2010. Heterogeneity among methicillin-resistant Staphylococcus aureus from Italian pig finishing holdings. Vet. Microbiol. 142:361-366.

Broens, E. M., E. A. M. Graat, P. J. van der Wolf, A. W. van der Giessen, E. van Duijkeren, J. A. Wahenaar, A. van Nes, D. J. Mevius, and M. C. M. de Jong. 2011. MRSA CC38 in the pig production chain. Prev. Vet. Med. 98:182-189.

CLSI. 2013a. VET01-A4 and VET01-S2. Performance Standards for Antimicrobial Disk and Dilution Susceptibility Tests for Bacterial Isolated from Animals; Approved Standard-Fourth Edition and Supplement, VET01A4E and VET01S2E. CLSI, Wayne, PA.

CLSI. 2013b. M02-A11 AND M100-S23. Performance Standards for Antimicrobial Disk Susceptibility Tests; Approved Standard-Eleventh Edition \& Performance Standards for Antimicrobial Susceptibility Testing; 23rd Informational Supplement. CLSI, Wayne, PA.

Cremonesi, P., T. Zottola, C. Locatelli, C. Pollera, B. Castiglioni, L. Scaccabarozzi, and P. Moroni. 2013. Identification of virulence factors in 16S-23S rRNA intergenic spacer genotyped Staphylococcus aureus isolated from water buffaloes and small ruminants. J. Dairy Sci. 96:7666-7674. 
DeLeo, F. R., M. Otto, B. N. Kreiswirth, and H. F. Chambers. 2010. Community-associated meticillin-resistant Staphylococcus aureus. Lancet 375:1557-1568.

EFSA. 2010. Scientific report of EFSA. Analysis of the baseline survey on the prevalence of methicillin-resistant Staphylococcus aureus (MRSA) in holdings with breeding pigs, in the EU, 2008. Part B: Factor associated with MRSA contamination of holdings. EFSA J. 8:1597.

Enright, M. C., N. P. Day, C. E. Davies, S. J. Peacock, and B. G. Spratt. 2000. Multilocus sequence typing for characterization of methicillin-resistant and methicillin-susceptible clones of Staphylococcus aureus. J. Clin. Microbiol. 38:1008-1015.

Feingold, B. J., E. K. Silbergeld, F. C. Curriero, B. A. G. L. van Cleef, M. E. O. C. Heck, and J. A. J. W. Kluytmans. 2012. Livestock density as risk factor for livestock-associated methicillinresistant Staphylococcus aureus, the Netherlands. Emerg. Infect. Dis. 18:1841-1849.

Feßler, A., R. G. M. Olde Riekerink, A. Rothkamp, K. Kadlec, O. C. Sampimon, T. J. G. M. Lam, and S. Schwarz. 2012. Characterization of methicillin-resistant Staphylococcus aureus CC398 obtained from humans and animals on dairy farms. Vet. Microbiol. 160:77-84.

Friese, A., J. Schulz, L. Hoehle, A. Fetsch, B. A. Tenhagen, J. Hartung, and U. Roesler. 2012. Occurrence of MRSA in air and housing environment of pig barns. Vet. Microbiol. 158:129-135.

Haran, K. P., S. M. Godden, D. Boxrud, S. Jawahir, J. B. Bender, and S. Sreevatsan. 2012. Prevalence and characterization of Staphylococcus aureus, including Methicillin-Resistant Staphylococcus aureus, isolated from bulk tank milk from Minnesota dairy farms. J. Clin. Microbiol. 50:688-695.

Hososaka, Y., H. Hanaki, H. Endo, Y. Suzuki, Z. Nagasawa, Y. Otsuka, T. Nakae, and K. Sunakawa. 2007. Characterization of oxacillin-susceptible mecA-positive Staphylococcus aureus: A new type of MRSA. J. Infect. Chemother. 13:79-86.

Kirk, J. H. 1984. Programmable calculator program for linear somatic cell scores to estimate mastitis yield losses. J. Dairy Sci. 67:441443

Kreausukon, K., A. Fetsch, B. Kraushaar, K. Alt, K. Müller, V. Krömker, K.-H. Zessin, A. Käsbohrer, and B.-A. Tenhagen. 2012. Prevalence, antimicrobial resistance, and molecular characterization of methicillin-resistant Staphylococcus aureus from bulk tank milk of dairy herds. J. Dairy Sci. 95:4382-4388.

Kumar, V. A., K. Steffy, M. Chatterjee, M. Sugumar, K. R. Dinesh, A. Manoharan, S. Karim, and R. Biswas. 2013. Detection of oxacillinsusceptible mecA-positive Staphylococcus aureus isolates by use of chromogenic medium MRSA ID. J. Clin. Microbiol. 51:318-319.

Luini, M., P. Cremonesi, G. Magro, V. Bianchini, G. Minozzi, B. Castiglioni, and R. Piccinini. 2015. Short communication Methicillinresistant Staphylococcus aureus (MRSA) is associated with low within-herd prevalence of intra-mammary infections in dairy cows: Genotyping of isolates. Vet. Microbiol. 178:270-274

Merialdi, G., E. Galletti, S. Guazzetti, C. Rosignoli, G. Alborali, A. Battisti, A. Franco, P. Bonilauri, G. Rugna, and P. Martelli. 2013. Environmental methicillin-resistant Staphylococcus aureus contamination in pig herds in relation to the productive phase and application of cleaning and disinfection. Res. Vet. Sci. 94:425-427.

Molla, B., M. Byrne, M. Abley, J. Mathews, C. R. Jackson, P. Fedorka-Cray, S. Sreevatsan, P. Wang, and W. A. Gebreyes. 2012. Epidemiology and genotypic characteristics of methicillin-resistant Staphylococcus aureus strains of porcine origin. J. Clin. Microbiol. 50:3687-3693.

Murakami, K., W. Minamide, K. Wada, E. Nakamura, H. Teraoka, and S. Watanabe. 1991. Identification of methicillin-resistant strains of staphylococci by polymerase chain reaction. J. Clin. Microbiol. 29:2240-2244.

Nemeghaire, S., M. A. Argudín, F. Haesebrouck, and P. Butaye. 2014. Epidemiology and molecular characterization of methicillin-resistant Staphylococcus aureus nasal carriage isolates from bovines. BMC Vet. Res. 10:153.

Paterson, G. K., J. Larsen, E. M. Harrison, A. R. Larsen, F. J. Morgan, S. J. Peacock, J. Parkhill, R. N. Zadoks, and M. A. Holmes. 2012. First detection of livestock-associated meticillin-resistant Staphylococcus aureus CC398 in bulk tank milk in the United Kingdom, January to July 2012. Euro Surveill. 17:20337.

Shopsin, B., M. Gomez, S. O. Montgomery, D. H. Smith, M. Waddington, D. E. Dodge, D. A. Bost, M. Riehman, S. Naidich, and B. N. Kreiswirth. 1999. Evaluation of protein A gene polymorphic region DNA sequencing for typing of Staphylococcus aureus strains. J. Clin. Microbiol. 37:3556-3563.

Smit, L. A. M., F. van der Sman-de Beer, A. W. J. Opstal-van Winden, M. Hooiveld, J. Beekhuizen, I. M. Wouters, J. Yzermans, and D. Heederik. 2012. Q Fever and pneumonia in an area with a high livestock density: A large population-based study. PLoS ONE 7:e38843 http://dx.doi.org/10.1371/journal.pone.0038843.

Spohr, M., J. Rau, A. Friedrich, G. Klittich, A. Fetsch, B. Guerra, J. A. Hammerl, and B. A. Tenhagen. 2011. Methicillin-resistant Staphylococcus aureus (MRSA) in three dairy herds in Southwest Germany. Zoonoses Public Health http://dx.doi.org/10.1111/ j.1863-2378.2010.01344.x.

Spoor, L. E., P. R. McAdam, L. A. Weinert, A. Rambaut, H. Hasman, F. M. Aarestrup, A. M. Kearns, A. R. Larsen, R. L. Skov, and J. R. Fitzgerald. 2013. Livestock origin for a human pandemic clone of community-associated methicillin-resistant Staphylococcus aureus. MBio 4:e00356-13. http://dx.doi.org/10.1128/mBio.00356-13.

Tavakol, M., R. G. Riekerink, O. C. Sampimon, W. J. van Wamel A. van Belkum, and T. J. Lam. 2012. Bovine-associated MRSA ST398 in the Netherlands. Acta Vet. Scand. 54:28.

van Cleef, B. A. G. L., H. Graveland, A. P. J. Haenen, A. W. van de Giessen, D. Heederik, J. A. Wagenaar, and J. A. J. W. Kluytmans. 2011. Persistence of livestock-associated methicillin-resistant Staphylococcus aureus in field workers after short-term occupational exposure to pigs and veal calves. J. Clin. Microbiol. 49:1030-1033.

van den Broek, I. V. F., B. A. G. L. van Cleef, A. Haenen, E. M. Broens, P. J. van der Wolf, M. J. M. van den Broek, X. W. Huijsdens, J. A. J. W. Kluytmans, A. W. van de Giessen, and E. W. Tiemersma. 2009. Methicillin-resistant Staphylococcus aure$u s$ in people living and working in pig farms. Epidemiol. Infect. 137:700-708.

van Duijkeren, E., P. D. Hengeveld, M. Albers, G. Pluister, P. Jacobs, L. Heres, and A. W. van de Giessen. 2014. Prevalence of methicillin-resistant Staphylococcus aureus carrying mecA or mecC in dairy cattle. Vet. Microbiol. 171:364-367.

van Loo, I., X. Huijsdens, E. Tiemersma, A. de Neeling, N. van de Sande-Bruinsma, D. Beaujean, A. Voss, and J. Kluytmans. 2007. Emergence of methicillin-resistant Staphylococcus aureus of animal origin in humans. Emerg. Infect. Dis. 13:1834-1839.

Vanderhaeghen, W., T. Cerpentier, C. Adriaensen, J. Vicca, K. Hermans, and P. Butaye. 2010. Methicillin-resistant Staphylococcus aureus (MRSA) ST398 associated with clinical and subclinical mastitis in Belgian cows. Vet. Microbiol. 144:166-171.

Voss, A., F. Loeffen, J. Bakker, C. Klaassen, and M. Wulf. 2005. Methicillin-resistant Staphylococcus aureus in pig farming. Emerg. Infect. Dis. 11:1965-1966. 